\title{
Nationales Design?
}

\author{
Auf der Suche nach dem „Holland-Rad” 1900-1940
}

\author{
von Anne-Katrin Ebert
}

\begin{abstract}
Überblick
Das „Holland-Rad“ ist ein Beispiel für ein „,anonymes Design“. Auch wenn der eigentliche Urheber dieses Designs im Sinne einer Person nicht bekannt ist, erübrigt sich hiermit jedoch nicht die Frage nach dem Ursprung dieser speziellen Gestaltung des technischen Objekts. Der Gestaltung des Gegenstands liegen immer auch eine Idee und Form zugrunde, die dessen Eigenart begründen. Das Design ist weniger ästhetische Überformung als vielmehr integraler Bestandteil des Objekts. Beim Holland-Rad konvergieren somit designhistorische und technikgeschichtliche Betrachtungen in der Frage, wie sich die Eigenart dieses Fahrradmodells begründet. Der vorliegende Beitrag vertritt die These, dass sich in der Materialität des Holland-Rads die spezifische Bedeutung des Fahrrads in der niederländischen Gesellschaft manifestiert. Anhand einer Analyse der Entwicklung der niederländischen Fahrradproduktion und der Aneignungsprozesse des Fahrrads in der niederländischen Gesellschaft wird die wesentliche Rolle der Konsumenten verdeutlicht, die als Ko-Konstrukteure und Ko-Designer maßgeblichen Einfluss auf die Gestalt technischer Artefakte ausübten.
\end{abstract}

\begin{abstract}
The „Dutch Bicycle“ is an anonymous design which cannot be attributed to one particular designer or producer. But this does not rule out an investigation into the origins of this particular form of technical artifact. At the basis of the particular shape of a technical artifact lies an idea which accounts for its characteristics. Design is not so much an aesthetic superimposition upon the object, but rather it is an integral part of the object itself. Design history and history of technology converge in the question posed in this article: why, and how, did the particular form of the „Dutch Bicycle“ came about? This article argues that the materiality of the „Dutch Bicycle“ manifests specific negotiations regarding the meaning and the function of the bicycle in Dutch society. By simultaneously analyzing the development of Dutch bicycle production and the appropriation of the bicycle in Dutch society, this article demonstrates the fundamental role of consumers as co-constructors and co-designers of technical artifacts.
\end{abstract}




\section{Einleitung}

Wer gegenwärtig unter Google den Begriff „Hollandrad“ eingibt, erhält 73.600 Treffer. Viele davon sind Verkaufsangebote von Holland-Rädern, die von niederländischen Marken wie Gazelle oder Sparta bis hin zum deutschen Kataloganbieter Otto reichen. Das Holland-Rad, so scheint es, ist ein Begriff, der im Deutschen mit einem spezifischen Design assoziiert wird. Es handelt sich beim Holland-Rad um ein nostalgisches „Cityrad“, ${ }^{1}$ traditionell in schwarz (obwohl andere Farben mittlerweile auch vertreten sind), mit einem vergleichsweise schweren Rahmen. Mit dem Holland-Rad verbunden ist eine aufrechte Sitzhaltung der Radfahrer.

Im Niederländischen existiert der Begriff des „Holland-Rads“ nicht, aber es gibt ein begriffliches Pendant, das so genannte „omafiets“ („Omafahrrad“) bzw. das „opoefiets“ („Opafahrrad“). Der Begriff „omafiets“ tauchte in den Niederlanden in den 1970er Jahren auf und bezeichnete ein schwarz gehaltenes Damenfahrrad mit tiefem Einstieg. Zwar findet sich auch im Deutschen die Tendenz, mit dem Holland-Rad ein Damenrad zu verbinden, aber im Niederländischen ist diese geschlechtliche Differenzierung deutlich stärker ausgeprägt.

Wie ein Holland-Rad resp. „omafiets“ ungefähr auszusehen hat, darüber gibt es einen weitgehenden, herstellerübergreifenden Konsens: Es hat zumeist einen Doppelrohrrahmen, bei dem die untere Stange gerade, die obere indes in einem leichten Bogen geführt wird, der „dames bocht“ („Damenbogen“). Gleichwohl ist dieses Design nicht auf einen bestimmten Designer zurückzuführen, sondern ein ,anonymes Design“ ${ }^{2}{ }^{2}$ Dies bedeutet jedoch nicht, dass die

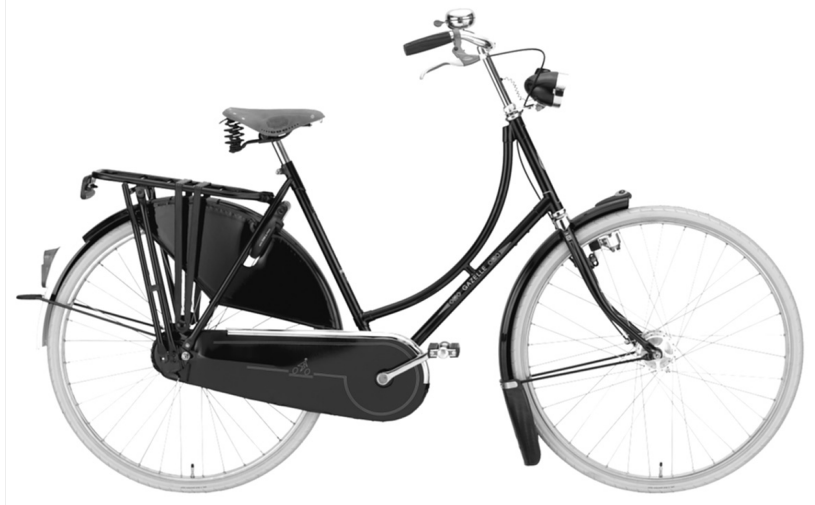

Abb. 1: Gazelle Toer Populair. Quelle: Gazelle GmbH.

1 Vgl. die Eingruppierung im Otto-Katalog, http://www.otto.de [Stand: 9.5.2009].

2 Vgl. Rolf Sachsse, Design, in: Leon R. Tsvasman (Hg.), Das Große Lexikon Medien und Kommunikation. Kompendium interdisziplinärer Konzepte, Würzburg 2007, S. 77f. Der 
Frage nach dem Ursprung dieser speziellen Gestaltung des technischen Objekts obsolet wäre. Auch wenn der Urheber des Designs nicht zu ermitteln ist, so liegen dem Gegenstand dennoch eine Idee und eine Form zugrunde, aus welchen heraus dessen Eigenart entstanden und zu verstehen ist.

Zuschreibungen, wie ein typisch niederländisches Fahrradmodell auszusehen habe, lassen sich - wie im Folgenden noch zu zeigen sein wird - bis in die 1920er Jahre zurückverfolgen. Mindestens ebenso lange galt es als selbstverständlich, dass die Niederlande ein Fahrradland seien. Gemeinhin wird dies mit der geographischen Beschaffenheit des Landes begründet. Es läge also nahe, hierin auch eine Ursache für die Herausbildung eines nationalen Designs beim Fahrrad zu vermuten. ${ }^{3}$ Tatsächlich ist die Bedeutung der naturräumlichen Bedingungen für die Nutzung des Fahrrads in den Niederlanden in einem aufwändigen Vergleich mehrerer europäischer Städte inzwischen relativiert worden. ${ }^{4}$ Die beiden niederländischen Technikhistoriker Frank Veraart und Adri Albert de la Bruhèze haben die Entwicklung des Fahrradgebrauchs in Amsterdam, Eindhoven, Enschede, Südost-Limburg, Antwerpen, Manchester, Kopenhagen, Hannover und Basel im 20. Jahrhundert analysiert und verdeutlicht, dass sich die Beliebtheit des Fahrrads in den Niederlanden nicht allein mit angeblich „objektiven“ Gründen wie geographischen oder morphologischen Gegebenheiten, also quasi außersystemisch, erklären lässt. Als entscheidenden Faktor ermittelten die beiden Autoren in ihrer Untersuchung die Bedeutung des Fahrrads in der Gesellschaft. ${ }^{5}$

Der vorliegende Beitrag stellt die These auf, dass sich diese Bedeutung, die die niederländische Gesellschaft dem Fahrrad beimaß, auch in der Materialität des Holland-Rads manifestierte. Somit ist das ,,anonyme Design“ des Holland-Rads weniger eine ästhetische Überformung vorhandener Fahrradmodelle als vielmehr integraler Bestandteil des Objekts. Design- und Technik-

Begriff des „anonymen Designs“ enthält mehrere Konnotationen. Er verweist auf die Anonymität des Designers, zugleich aber auch auf die weitreichende, alltägliche Präsenz und Akzeptanz des ,,anonymen Designs“ gerade auch im Kontrast zum Design bekannter Designer. Zum ,anonymen Design“ als historisches Problem s.a. Hazel Conway (Hg.), Design History: A Student's Handbook, London 2002, S. 8; zum ,anonymen Design“ des Massenkonsums vgl. Gert Selle, There is No Kitsch, There is Only Design, in: Victor Margolin (Hg.), Design Discourse: History, Theory, Criticism, Chicago u. London 1989, S. 55-67, hier S. 56.

3 Auf die Bedeutung geographischer Bedingungen bei der ,social construction of technology" wies auch schon hin: Thomas P. Hughes, The Evolution of Large Technological Systems, in: Wiebe E. Bijker, Thomas P. Hughes u. Trevor Pinch (Hg.), The Social Construction of Technological Systems: New Directions in the Sociology and History of Technology, 7. Aufl. Cambridge, MA 1999, S. 51-83, hier S. 70.

4 A.A. de la Bruhèze u. F.C.A. Veraart, Fietsverkeer in praktijk en beleid in de twintigste eeuw. Overeenkomsten en verschillen in fietsgebruik in Amsterdam, Eindhoven, Enschede, Zuidoost-Limburg, Antwerpen, Manchester, Kopenhagen, Hannover en Basel, Den Haag 1999.

5 Ebd., S. 27ff. u. 196ff. 
geschichte konvergieren in der Frage, wieso es zur Produktion und Konsumtion von eben diesem spezifischen Fahrradmodell in den Niederlanden kam. Hierin überschneiden sich nicht nur Technik- und Designgeschichte, sondern auch Konsum- und Produktionsgeschichte: Die Herausbildung eines spezifisch niederländischen Fahrradmodells tangiert sowohl die Frage nach einem nationalen Technikstil in der Produktion ${ }^{6}$ als auch die nach der Rolle der Konsumenten als Ko-Konstrukteure und Ko-Designer eines technischen Artefakts. ${ }^{7}$ Im Folgenden soll diesen Aspekten sowohl anhand der Entwicklung der Fahrradproduktion in den Niederlanden als auch anhand einer eingehenden Analyse der Aneigungsprozesse des Fahrrads seitens der Konsumenten nachgegangen werden.

\section{Das moderne Industrieprodukt: Aneignungsprozesse des Fahrrads im niederländischen Bürgertum um 1900}

Radfahren war in den Niederlanden um 1900 noch weitestgehend ein Sport. Mit dieser Praxis verband sich zugleich ein gehobener, luxuriöser Lebensstil. Wer Sport trieb, verfügte über die freie Zeit und das nötige Geld, sich dies leisten zu können. Wer Sport trieb, war ein Mann von Welt, kannte die englische Praxis und ließ sich auch nicht von der mit englischen Fachtermini durchsetzten Sprache des Sports abschrecken. Wer Sport trieb, war Mitglied einer elitären Eigenwelt. Diese distinktive Funktion war zunächst auch im „Bicyclesport" angelegt. Die Mitgliedschaft in den Clubs wurde mit Hilfe der Ballotage kontrolliert und auf einen kleinen, elitären Kreis beschränkt. Die „Clubs“ entfalteten ein lebhaftes Vereinsleben mit gemeinsamen Radausflügen, Wettrennen und Geschicklichkeitsübungen bis hin zu winterlichen Theateraufführungen und Ballabenden. ${ }^{9}$

6 Zu nationalen Technikstilen vgl. Joachim Radkau, Technik in Deutschland. Vom 18. Jahrhundert bis zur Gegenwart, Frankfurt a.M. 1989, S. 31-40. Zur Entwicklung nationaler Technikstile allgemein s.a. Eda Kranakis, Constructing a Bridge: An Exploration of Engineering Culture, Design, and Research in Nineteenth-Century France and America, Cambridge, MA 1997; Gabrielle Hecht, The Radiance of France: Nuclear Power and National Identity after World War II, Cambridge, MA 1998.

7 Zur Bedeutung der Konsumenten als Ko-Konstrukteure in der Technikgeschichte s. v.a. Nelly Oudshoorn u. Trevor Pinch (Hg.), How Users Matter: The Co-Construction of Users and Technologies, Cambridge, MA u. London 2004.

8 Zur Geschichte des Fahrrads und des Radfahrens allgemein s. Andrew Ritchie, King of the Road: An Illustrated History of Cycling, Londen 1975; Pryor Dodge, The Bicycle, Paris 1996; Glen Norcliffe, The Ride to Modernity: The Bicycle in Canada, 1869-1900, Toronto u.a. 2001; D.V. Herlihy, Bicycle: The History, New Haven, CT 2004; Peter Cox, Dave Horton u. Paul Rosen (Hg.), Cycling and Society, Aldershot 2007; für Deutschland s. Volker Briese, Wilhelm Matthies u. Gerhard Renda (Hg.), Wegbereiter des Fahrrads, Bielefeld 1997; Rüdiger Rabenstein, Radsport und Gesellschaft. Ihre sozialgeschichtlichen Zusammenhänge in der Zeit von 1867 bis 1914, 2. Aufl. Hildesheim, München u. Zürich 1995.

9 Vgl. Algemeene Nederlandsche Wielrijders-Bond (A.N.W.B.) Archief 192, T.J. Klaverweiden, Een Wielrijders-Oase in de Kalverstraat en nog wat, o.O. o.J. [1897]. 
Soziale Distinktion fand ihren Ausdruck auch in der durch modernste Stahlverarbeitung geschaffenen Materialität der Fahrräder selbst. Die Bedeutung des Werkstoffs schlug sich sowohl in der niederländischen als auch in der deutschen Sprache mit Wortschöpfungen wie ,het stalen ros“ bzw. dem „Stahlross" nieder. Mit regem Interesse verfolgten Radfahr-Zeitschriften und Handbücher die Produktion der Räder und diskutierten und bewerteten die nationale Industrie gegenüber der internationalen, d.h. vor allem der englischen Konkurrenz. ${ }^{10}$

Das Fahrrad aus Stahlrohr, wie es zuerst in Coventry und England hergestellt wurde, verkörperte das moderne Industrieprodukt. Die Stabilität und Unverwüstbarkeit des Stahls ging beim Fahrrad einher mit den Errungenschaften moderner Leichtbauweise. Gerade auch in der Konstruktion der Räder und Speichen folgten die Fahrradmodelle dem technischen Trend der Zeit. Die kombinierte Zug- und Druckbeanspruchung, die in den industriellen Bauten, beispielsweise von Hallen und Brücken, die Druck- und Biegebeanspruchung traditioneller Bauweisen ablöste, fand sich auch im Fahrrad wieder beim Übergang von der Radial- zur Tangentialspeiche. ${ }^{11}$ In Aufbau und Material vereinigte das Fahrrad die Leichtigkeit der Zugtechnik mit der hohen Festigkeit des Stahls. Es war Ausdruck modernster Ingenieurskunst und Präzisionstechnik und vermittelte den Radfahrern eine Erfahrung auf dem neuesten Stand der Technik. Die niederländische Fahrradindustrie spielte um die Jahrhundertwende allerdings eine geringe Rolle auf dem heimischen Markt: ${ }^{12}$ es dominierten englische und deutsche Produkte.

1883 hatte sich ein niederländischer Dachverband gegründet, der seit 1885 unter dem Namen „Algemeene Nederlandsche Wielrijders-Bond“(A.N.W.B.) agierte. Der Verband hob insbesondere die Bedeutung des Fahrrads als Erziehungsmittel und zur Charakterformung hervor, mit der er nicht nur die gesellschaftliche Relevanz des Radfahrens sondern auch die der Radfahrer betonte. Sein langjähriger Vorsitzender, Edo Bergsma, pries den Segen, den das Radfahren in körperlicher, gesellschaftlicher und auch sittlicher (,zedelijker") Hinsicht darstellen könne. ${ }^{13}$ In einer Rede anlässlich des zehnjährigen Bestehens des A.N.W.B. betonte er, das Radfahren sei ein männlicher, gesunder Sport, der die Muskeln stähle, den Blick erweitere und den Geist gesund halte beim „Kern der Nation“. ${ }^{14}$

10 Vgl. A.J. Werten u. W. van Sas, Handboek en Vraagbaak voor den Wielrijder, bevattende nuttige Wenken en Raadgevingen, aan de Practijk ontleend, Cuyk a/d Maas 1894, S. $15 \mathrm{ff}$.

11 Vgl. Joachim Krausse, Das Fahrrad. Von der ,kindischen“ Kombinatorik zur Montage, in: Wolfgang Ruppert (Hg.), Fahrrad, Auto, Fernsehschrank. Zur Kulturgeschichte der Alltagsdinge, Frankfurt a.M. 1993, S. 79-118, hier S. 103.

12 Vgl. H. Meijer, Holland, de grootste afnemer van Engelsche rijwielen, in: De Kampioen 24, 1907, S. 939f

13 E. Bergsma, Openingsrede ter gelegenheid van de Algemeene Vergadering, in: De Kampioen 9, 1892, S. 1023f., hier S. 1023.

14 Ders., De Bondsfeesten, in: De Kampioen 10, 1893, S. 713 ff., hier S. 714. 
Begriffe wie „Männlichkeit" und „Sittlichkeit“ hatten eine politische Konnotation, es waren zentrale Kampfbegriffe einer seit den 1870ern in den Niederlanden geführten politischen Auseinandersetzung zwischen dem liberalen Establishment und neuen sozialen Gruppierungen. Religiöse Gruppierungen, wie die „Anti-Revolutionären“ von Abraham Kuyper und die Katholiken stellten die seit der Verfassungsreform von Johan Rudolf Thorbecke von 1848 existierende Vormachtstellung der Liberalen in Frage. Indem Politiker wie Abraham Kuyper für einen Zugang der „kleinen Leute“ (im Niederländischen „kleine luyden“) zur Politik plädierten, attackierten sie auch die bisherigen Vorstellungen und Konventionen darüber, welche Eigenschaften einen niederländischen Bürger zur Teilnahme an der Politik qualifizierten. Die Auseinandersetzungen über eine Erweiterung des Wahlrechts waren mithin auch Kontroversen darüber, wie sich niederländische (Staats-)Bürger $\mathrm{zu}$ verhalten hatten und wie der politische Stil im Lande auszusehen hatte. ${ }^{15}$ Politiker wie Kuyper oder der Sozialist Domela Nieuwenhuis sorgten mit ihrem emotionalen Stil für viel Aufsehen. Demgegenüber appellierte die alte liberale Elite vor allem an „Männlichkeit“ und „Sittlichkeit“. In diesem Kontext hatte der Begriff „mannelijk“ vor allem die Konnotation von ,erwachsen“, ,ausgeglichen“, ,selbstbeherrscht“, ,unabhängig“, „standhaft" und „willensstark“. ${ }^{16}$ Mit der ,Zedelijkheid“ verbanden liberale Politiker vor allem Konzepte von Kontrolle und Selbstbeherrschung. ${ }^{17}$

Im Kontext dieser Auseinandersetzungen zwischen liberalem Bürgertum und neuen sozialen Gruppierungen lässt sich auch die Arbeit des niederländischen Radfahrerverbands A.N.W.B. sehen. Der A.N.W.B. war ein liberaler Verband und deutete die körperliche Beherrschung, die für das Radfahren notwendig war, als politische Lektion. Mit dem Fahrrad lerne das Bürgertum - der „Kern der Nation“ - liberale Tugenden wie Selbstbewusstsein und Unabhängigkeit. Der Sport wurde ,een uitstekende oefening voor toekomstige mannen van zaken of politici in den dop". ${ }^{18}$

Schwerpunkt der Tätigkeit des A.N.W.B. war die Förderung des Radtourismus, den der Verband als die Möglichkeit erkannte, den Bürgern die eigene Nation nahe zu bringen. Der A.N.W.B. konstruierte und normierte hierbei

15 I. de Haan u. H. te Velde, Vormen van politiek. Veranderingen van de openbaarheid in Nederland 1848-1900, in: Bijdragen en mededelingen betreffende de geschiedenis der Nederlanden 111, 1996, S. 167-200.

16 Vgl. H. te Velde, Viriliteit en Opoffering. ,Mannelijkheid' in het Nederlandse politieke debat van het fin-de-siècle, in: Groniek 29, 1996, S. 278-291 [zuvor erschienen in: Tijdschrift voor Vrouwenstudies 56, 1993, S. 421-433].

17 Vgl. ders., Zedelijkheid als ethiek en seksueel fatsoen. De geschiedenis van een Nederlands begrip, in: R. Aerts u. K.van Berkel (Hg.), De pijn van Prometheus. Essays over cultuurkritiek en culturpessimisme, Groningen 1996, S. 198-218.

18 W. J. H. Mulier, Iets over sport en over de sporttentoonstelling te Scheveningen, in: Eigen Haard 29, 1892, S. 459-462, hier S. 461. Übersetzung: „eine herausragende Übung für zukünftige Geschäftsleute oder künftige Politiker“. 
mit seinen Reiseführern, Reiseberichten und Bildbänden für Radfahrer nachhaltig das Bild von den Niederlanden. ${ }^{19} 1888$ veröffentlichte er seinen ersten Reiswijzer, ein Handbuch mit Kartenmaterial, in dem die wichtigsten Radtouren durch das Land verzeichnet und beschrieben waren. Urheber war der Engländer Charles Bingham, der das Land für den niederländischen Radfahrerverband in Kartenform brachte und dabei vor allem auf seine eigenen und die Erfahrungen anderer Mitglieder des A.N.W.B. auf dem Rad zurückgriff. ${ }^{20}$

Mit dem Reiswijzer machte der A.N.W.B. erste Vorgaben, wie der nationale Raum von den Radfahrern erschlossen werden sollte. Weit mehr als ein Handbuch für Wege, Übernachtungs- und Reparaturmöglichkeiten, zeigte der Reiswijzer an, welche Strecken, Orte und Landschaften als besichtigungswert angesehen wurden und erfasste so die Niederlande erstmals als nationales Ganzes für die Radfahrer. Aus Sicht des A.N.W.B. konnte ein Kennenlernen des eigenen Landes nur sinnvoll auf der Basis der Lektüre des Reiswijzer erfolgen, erklärte 1907 der Chefredakteur der Verbandszeitschrift De Kampioen, Henri Meijer. Alles andere sei planloses Vorbeifahren an der Schönheit und den Sehenswürdigkeiten des Landes. ${ }^{21}$

Das planlose Fahren im Land hatte der A.N.W.B. seit 1894 auch durch das Aufstellen von Wegweisern einzudämmen versucht. ${ }^{22}$ Der Verband sorgte selbst für die Finanzierung, Herstellung und Verwaltung der Wegweiser, vor allem aber setzte er dem System der Gemeinde-, Provinzial- und Reichswege ein Wegweisesystem entgegen, das sich ausschließlich am nationalen Raum der Niederlande orientierte und keine Beschränkung auf Gemeinden oder Provinzen zuließ. ${ }^{23}$ Die Erschließung der Landstraßen in den Niederlanden durch ein nationales Wegweisersystem erfolgte lange Zeit ausschließlich auf Initiative des Radfahrerverbands. Erst nach dem Ersten Weltkrieg begann der niederländische Staat, sich an diesem Projekt finanziell zu beteiligen. ${ }^{24}$

19 Zur Diskussion über den Tourismus und den „tourist gaze“ s. John Urry, The Tourist Gaze: Leisure and Travel in Contemporary Societies, London 1990; Rudy Koshar, ,What Ought to Be Seen': Tourists' Guidebooks and National Identities in Modern Germany and Europe, in: Journal of Contemporary History 22, 1998, S. 323-340. Eine gute Zusammenfassung bieten Shelley Baranowski u. Ellen Furlough, Introduction, in: diess. (Hg.), Being Elsewhere: Tourism, Consumer Culture and Identity in Modern Europe and North America, Ann Arbor 2001, S. 1-31.

20 J.G. Kikkert, Op stap in Nederland. Toerisme vroeger en nu, Weesp 1985, S. 56.

21 Vgl. H. Meijer, De Reiswijzer van den A.N.W.B., in: De Kampioen 24, 1907, S. $717 \mathrm{ff}$.

22 Vgl. C. Versteeg, 100 jaar ANWB-bewegwijzering, Den Haag 1994, S. 6. Seit 1892 hatte der Bund Warnschilder bei gefährlichen Abfahrten aufgestellt.

23 Neben der Behinderung von Radfahrern auf den Wegen entspannte sich ein größerer Konflikt zwischen Verband und Landbevölkerung um diese Wegweiser. Das fortwährende Abholzen der Leitschilder und -pfosten diente vermutlich nicht nur der Brennholzbeschaffung für den Winter sondern war auch Ausdruck der Weigerung, das eigene Dorf oder die eigene Region den national orientierten Radfahrern zu öffnen. Vgl. D. Schaap (Hg.), Een eeuw wijzer, Den Haag 1983, S. 166.

24 Ebd., S. 166ff. 
Neben den Reiseführern war die Fotografie ein wesentliches Mittel bei der Konstruktion des Bildes vom eigenen Land. Zwischen 1908 und 1910 veröffentlichte der A.N.W.B. die vierbändige Bildband-Reihe Ons eigen land. Die Bildbände erschienen anlässlich des 25-jährigen Geburtstags des Verbands. Die Fotografien in den aufwändig gestalteten Alben waren eine Blütenlese des A.N.W.B. aus über 1.000 Fotos. Angesichts dieser Aufnahmen, vermutete Chefredakteur Henri Meijer, würde so mancher Besitzer eines Fahrrads beschämt sein, dass er so viel Schönheit in seinem unmittelbaren Umfeld bisher nicht gekannt habe. ${ }^{25}$ Der Erfolg überstieg die Erwartungen des A.N.W.B. Innerhalb eines halben Jahres war der erste Band ausverkauft, so dass der Verband bereits im März 1909 eine zweite Auflage herausgab.

Das Engagement des niederländischen Radfahrerverbands für den Radtourismus erfolgte auch vor dem Hintergrund der wachsenden Skepsis gegenüber den Radrennen. Die Wettrennen stellten ursprünglich ein weiteres Tätigkeitsfeld des Verbands dar, galten aber bereits in den 1880er Jahren als Nebenschauplatz, während das Hauptaugenmerk auf der Verbesserung des Radtourismus lag. Im November 1887 wurde in einem Artikel in De Kampioen davor gewarnt, dass die vielen Wettrennen mit ihren Diners, Soiréen und Amüsements zwar eine angenehme Entspannung von der täglichen Arbeit seien, dass aber darin keineswegs das Hauptziel des Radsports gesehen werden dürfe. Dieses sei vielmehr, Ausflüge zu unternehmen und die Sehenswürdigkeiten des Landes besser kennen zu lernen. ${ }^{26} \mathrm{Als}$ sich gegen Ende des 19. Jahrhunderts die Unregelmäßigkeiten auf der Rennbahn häuften, entschied sich der niederländische Verband zu einer radikalen Maßnahme: Gegen den Widerstand derjenigen, die den Verband dazu verpflichten wollten, die Moral des Sports auch weiterhin im Rennsport-Bereich zu schützen, beschloss die außerordentliche Allgemeine Versammlung des A.N.W.B. vom 27. November 1898, sich aus dem Rennsport vollständig zurückzuziehen und künftig allein um das Touren zu kümmern. ${ }^{27}$ Die Entscheidung entsprang vor allem der Sorge, der Bund könnte mit einem weiteren Engagement im korrupten professionellen Wettkampfsport seinen Ruf und das Ansehen des Radfahrens dauerhaft beschädigen. ${ }^{28}$

25 H. Meijer, Ons eigen land, in: De Kampioen 25, 1908, S. 317f., hier S. 317.

26 Ohne Verfasser [o.V.], Es war ein Traum, in: De Kampioen 4, 1887, S. 255f., hier S. 256. Vgl. a. o.V., Veertig jaar. Uit het Archief der Kampioen-Redactie, o.O. 1923, S. 58-61.

27 Vgl. o.V., Loslaten of niet, in: De Kampioen 14, 1897, S. 489ff. Vgl. auch die Debatte um die Aufgabe des Rennsports im Vorstand des A.N.W.B., die M. F. A. Linders-Rooijendijk, Gebaande wegen voor mobiliteit en vrijetijdsbesteding, [I]: De ANWB als vrijwillige associatie 1883-1937, Heeswijk 1989, S. 85-90, detailliert wiedergibt; s. darüber hinaus a. Schaap (wie Anm. 23), S. 40ff.

28 Vgl. hierzu die düstere Abrechnung mit dem Radrennsport bei J.C. Burkens, Wielrijden, in: J. Feith (Hg.), Het boek der sporten, Amsterdam 1900, S. 225-260, bes. S. 230f., S. 234, S. 252, S. 255. 
Der Verbandsbeschluss hatte große Auswirkungen auf die Praxis des Radfahrens und die Gestalt der Fahrräder in den Niederlanden allgemein. 1904 konstatierte die Fahrradzeitschrift De Fiets, dass sich das Rennwesen im Land nach der Saison 1899/1900 nie wieder ganz erholt habe. ${ }^{29}$ Einen weiteren schweren Schlag erlitten die Straßenrennen darüber hinaus durch die Einführung des ersten nationalen Straßenverkehrsgesetzes von 1905.

Das „Motor- und Rijwielwet“ von 1905 sah in Paragraph 14 ein grundsätzliches Verbot von Wettrennen auf öffentlichen Straßen vor. ${ }^{30}$ Ohne dass die Rolle des A.N.W.B. bei dieser Regelung genau zu bemessen wäre, kam das Gesetz den wiederholten energischen öffentlichen Erklärungen des Verbands eindeutig entgegen. ${ }^{31}$ Der Wettkampfsport, die zunehmende Kommerzialisierung der Radrennen und die Raserei auf den Straßen gefährdeten aus Sicht der Verantwortlichen im A.N.W.B. das bewusste „Erfahren“ des eigenen Landes. In Frankreich war 1902 mit der „Tour de France“ ein spektakuläres, kommerzielles Rennen ins Leben gerufen worden, das die verschiedenen Provinzen und regionalen Unterschiede thematisierte und in einer Rundfahrt zusammenführte. In den Niederlanden paralysierte das „Motor- en Rijwielwet" von 1905 endgültig den Radrennsport im Lande, der schon unter dem Ausstieg des A.N.W.B. aus dem Radrennsport schwer gelitten hatte.

Der A.N.W.B. machte keinen Hehl aus seiner vehementen Ablehnung des Rennsports sowie insbesondere der Straßenrennen, die unmittelbar das Terrain des Touristenbundes berührten. Das Handbuch des Verbands von 1900 vermerkte triumphierend: „De Algemeene Nederlandsche Wielrijders-Bond [...] bevordert uitsluitend het toerisme“ und „heeft door zijn invloed de wegwedstrijden zooveel mogelijk doen beperken". ${ }^{32}$ Der Abschied vom Rennwesen war, so bemerkte ein Artikel in De Kampioen rückblickend 25 Jahre später, eine der gewichtigsten, wenn nicht die wichtigste Entscheidung in der Geschichte des A.N.W.B. ${ }^{33}$ Der Verband konzentrierte sich fortan ausschließlich auf den Radtourismus und die zunehmende alltägliche Nutzung des Fahrrads im Straßenverkehr.

29 O.V., Wielerwedstrijden, in: De Fiets 372, 1904, S. 1f.

30 Vgl. M.J. Adriani Engels, Van Jaap Eden tot Jan Derksen. De wielersport in Nederland gedurende tachtig jaar, Amsterdam 1947, S. 36.

31 Jan de Bas führt das Verbot von Radrennen im Verkehrsgesetz unmittelbar auf die orthodox-protestantische Ausrichtung der Anti-Revolutionären Partei zurück, die gemeinsam mit den Katholiken im Kabinett Kuypers das Verkehrsgesetz beschlossen hatten. Zugleich aber waren die Straßenrennen auch nicht im Interesse des liberalen A.N.W.B., so dass sich hier aus unterschiedlichen Gründen eine sehr breite Allianz gegen die Radrennen formiert hatte; vgl. J. de Bas, Hervormd op wielen. Wielerkampioen Cees Erkelens, Soesterberg 2005, S. 37f.

32 Handboekje 1900 van den Algemeenen Nederlandschen Wielrijders-Bond, 's Gravenhage 1900, S. 2f. Übersetzung: „Der Allgemeine Niederländische Radfahrerbund [...] befördert ausschließlich den Tourismus [...] und hat durch seinen Einfluss die Straßenrennen so weit wie möglich eingedämmt“".

33 O.V., De A.N.W.B. en het Congres der U.C.I., in: De Kampioen 42, 1925, S. 855 f. 


\section{Das Fahrrad als Massenkonsumgut und Mittler „niederländischer“ Tugenden}

Ab 1900 verbreitete sich das einstmalige Luxusgut Fahrrad zunehmend in der niederländischen Bevölkerung. Ein wesentlicher Faktor war hierbei der rasante Preisverfall durch neue Produktionsmethoden. Anhand der Fahrradkataloge der niederländischen Firma Fongers lässt sich diese Preisentwicklung nachverfolgen. Das teuerste Qualitätsrad der Firma war das Damenrad ,Soort A“, das 1898 für 212,50 Gulden von Werksseite angeboten wurde. Der Preis sank bis auf 158 Gulden im Jahr 1915. ${ }^{34}$ Das billigste Herrenrad der „Soort C" wurde 1898 für 110 Gulden angeboten, 1914 war das billigste Fahrrad im Angebot von Fongers, das „Holland Herenfiets“, schon für 55 Gulden zu haben. Bei den billigen Herren-Modellen verzeichnete der niederländische Fahrradproduzent somit zwischen 1898 und 1914 einen Preisrückgang um genau die Hälfte. Das Durchschnittseinkommen in den Niederlanden lag um 1910 in den Sektoren Agrar, Industrie und Dienstleistung bei monatlich 44 Gulden, 53 bzw. 60 Gulden. Ein Fahrrad kostete also immer noch ungefähr ein durchschnittliches Monatseinkommen. Dies war jedoch im Vergleich zu den 1880er Jahren ein erheblicher Preisrückgang: Damals kostete ein Hoch-

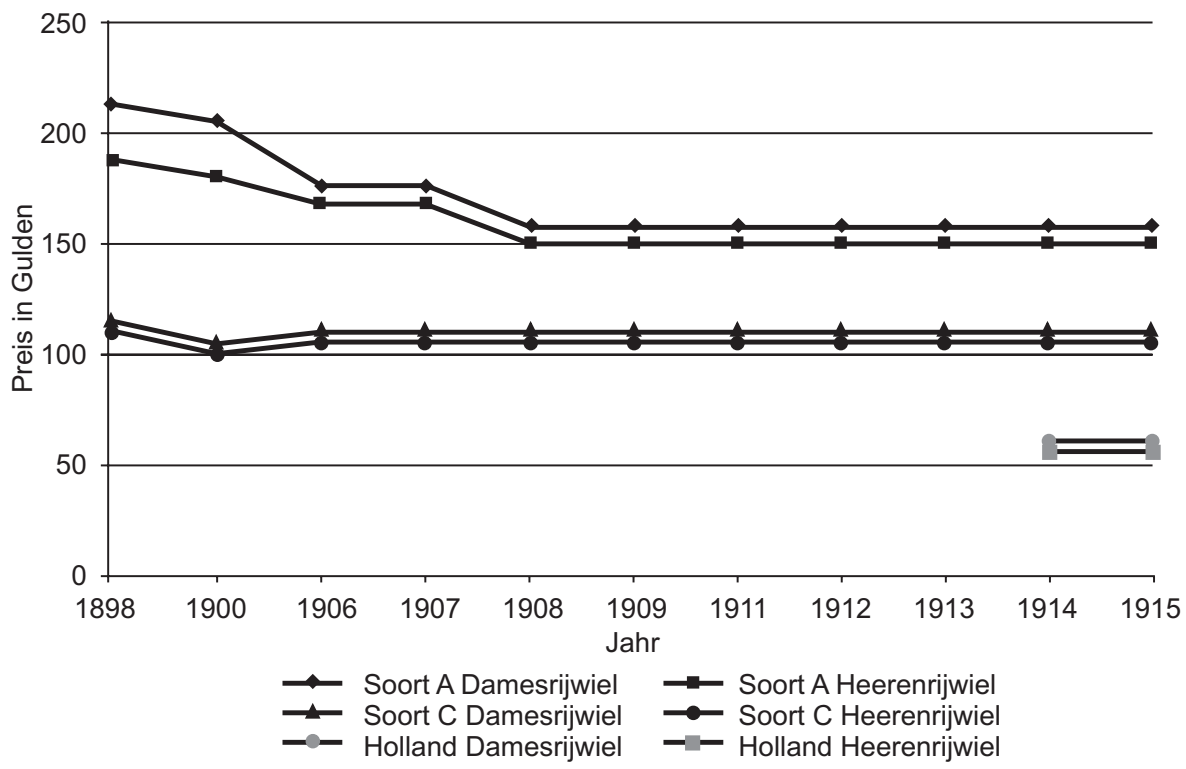

Graf. 1: Preisentwicklung Fongers. Quelle: Groninger Archieven 1300, Archieven van de Groninger Rijwielenfabriek A. Fongers, Inv. nrs. 231 und 232, Prijslijsten en prijscouranten 1898-1966.

34 Vgl. Groninger Archieven 1300, Archieven van de Groninger Rijwielenfabriek A.Fongers, Inv. nrs. 231 und 232, Prijslijsten en prijscouranten 1898-1966. 
rad das Vier- bis Achtfache der monatlichen Durchschnittseinkommen in den drei Sektoren. ${ }^{35}$

Der Preisverfall bei Fahrrädern war ein internationales Phänomen, ${ }^{36}$ die Verbreitung des Fahrrads in den Niederlanden wurde hingegen zunehmend als nationale Besonderheit wahrgenommen. Zwei Fahrradsteuern ermöglichen einen Einblick in die allmähliche Diffusion des Fahrrads in der Bevölkerung. Die erste Fahrradsteuer, die an die Besteuerung des Wohnraums gekoppelt war, existierte von 1899 bis 1912, die zweite wurde zwischen 1924 und 1941 erhoben. 1899 waren insgesamt 94.370 Fahrräder in den Niederlanden steuerlich erfasst. ${ }^{37}$ Bei einer Bevölkerung von 5.074.632 Einwohnern bedeutete dies, dass statistisch gesehen $1,86 \%$ bzw. jede 53ste Person ein Fahrrad besaß. In den darauf folgenden Jahren stieg die Anzahl der besteuerten Fahrräder kontinuierlich an. ${ }^{38}$ Setzt man diese in Verhältnis zur wachsenden Bevölkerungszahl des Landes, so ergibt sich eine Steigerung von 2\% im Jahr 1900 über 5\% im Jahr 1905 auf 9\% im Jahr 1910. 1912 erteilte die niederländische Steuerbehörde für 646.925 Fahrräder Steuerbescheinigungen, dies betraf $11 \%$ der Bevölkerung.

Insgesamt sind diese Zahlen jedoch mit einer gewissen Vorsicht zu genießen; selbst von staatlicher Seite ging man fest davon aus, dass die Steuerhinterziehung beim Fahrrad besonders groß war. Verlässlicher erscheinen hingegen die Zahlen der Fahrradsteuer ab 1924, die über eine Plakette am Fahrrad funktionierte. Mit Straßenkontrollen konnte deren Zahlung wesentlich effektiver kontrolliert werden. Bereits im ersten Steuerjahr kam ein Fahrrad auf jede vierte Person in den Niederlanden, ${ }^{39}$ am Ende der 1920er Jahre auf jede dritte. Zehn Jahre später betrug die Anzahl der besteuerten Fahrräder im Land im Verhältnis zur Gesamtbevölkerung 43\%. Statistisch gesehen verfügte 1940 also beinahe jede zweite Person über ein eigenes Rad.

35 Vgl. L. Soltow u. J.L. van Zanden, Income and Wealth Inequality in the Netherlands 16th-20th Century, Amsterdam 1998, S. 157, Tabelle 7.4.

36 Vgl. hierzu z.B. die Preisentwicklung in Deutschland bei A. Lepper, Die Entwicklung und Lage der Bielefelder Naehmaschinen- und Fahrradindustrie bis zum Jahre 1914, ihre Stellung innerhalb der deutschen Fachindustrie unter besonderer Beruecksichtigung der Konjunktur, Dissertation an der Universität Greifswald 1922, S. 161; s.a. A.E Harrison, The Competitiveness of the British Cycle Industry, 1890-1914, in: Economic History Review 22, 1969, S. 287-303.

37 O.V., Welsprekende Cijfers, in: De Kampioen 18, 1901, S. 211 ff., hier S. 212.

38 O.V., De nijverheid in 1912, in: De Kampioen 30, 1913, S. 756-757; vgl. a. o.V., Het aantal rijwielen in Nederland, in: De Kampioen 28, 1911, S. 643ff.; und o.V., Statistiek, in: De Kampioen 32, 1915, S. 44; vgl. a. o.V., De opkomst der rijwielen en de rijwielindustrie, in: De Kampioen 29, 1912, S. 984f. Die Bevölkerungszahlen der Niederlande nach Historie bevolking, huishoudens en bevolkingsontwikkeling unter http:// statline.cbs.nl/StatWeb [Stand: 30.8.2009].

39 Vgl. F.H.M. Grapperhaus, Over de loden last van het koperen fietsplaatje. De Nederlandse rijwielbelasting 1924-1941, Franeker 2006, S. 65. 
Ab den 1920er Jahren etablierte sich bereits die Verbindung des Fahrrads mit den Niederlanden in der in- und ausländischen Presse. 1921 berichtete die Londoner Zeitung The Times in ihrer „Dutch Number“: „One of the features of the Netherlands which strikes the foreign visitor most forcibly is

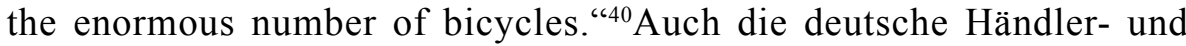
Produzentenzeitschrift Radmarkt berichtete vom Fahrradverkehr im Nachbarland unter dem Titel „Eine Nation auf Rädern“. ${ }^{41}$ In der in Boston erscheinenden Zeitschrift The Christian Science Monitor hieß es 1922, dass es kein anderes Land auf der Welt gäbe, wo so viele Bewohner Rad fuhren wie in den Niederlanden. ${ }^{42}$ Auf einer amerikanischen Autokarte der Firma Rand Mc.Nally \& Comp., bei der die einzelnen Länder und Städte Europas mit kleinen Zeichnungen berühmter Baudenkmäler und Sehenswürdigkeiten angedeutet wurden, war bei den Niederlanden ein Radfahrer eingezeichnet. ${ }^{43}$ Nicht zuletzt feierte auch der A.N.W.B., der seit 1905 den Zusatz „Toeristenbond voor Nederland" führte, anlässlich seines 40-jährigen Jubiläums 1923 und seines 50-jährigen Jubiläums 1933 das ,fietsland Nederland“. ${ }^{44}$

Auf die zunehmende Verbreitung des Fahrrads in der Bevölkerung reagierte der einstmals elitäre liberale Verband mit einem Politikwechsel: Es ging nicht mehr länger darum, die Interessen der eigenen Kreise zu bewahren, sondern die eigenen Werte der gesamten Bevölkerung zu vermitteln. Dieser Wandel des A.N.W.B. wird verständlich vor dem Hintergrund der gesellschaftlichen Veränderungen. Mit der Erweiterung des Wahlrechts 1896, der Einführung des vollen Männerwahlrechts 1917 sowie schließlich der Einführung des allgemeinen Wahlrechts für Männer und Frauen 1920 bemühten sich zahlreiche unterschiedliche liberale Gruppen im Land um eine Erziehung dieser „Masse“ der Bevölkerung zu verantwortungsvollen Bürgern. ${ }^{45}$ Der A.N.W.B. kann hierbei durchaus in die Tradition jener bürgerlich-liberalen Initiativen des 19. Jahrhunderts gestellt werden, die in der nie-

40 G.A. Pos, Cycling. For Business and Pleasure: Ideal Road Conditions, in: The Times, „The Dutch Number“ vom 6. Dezember 1921, S. 15.

41 O.V., Eine Nation auf Rädern, in: Der Radmarkt 1530, 1920, S. 13.

42 Vgl. die Zusammenfassung des Artikels in The Christian Science Monitor in: o.V.,Wielrijden in Nederland, in: De Kampioen 39, 1922, S. 1026.

43 Vgl. o.V., Nederland,...het heilige fietsen-land, in: De Kampioen 44, 1927, S. 1107.

44 Vgl. Veertig jaar (wie Anm. 26), S. 2; Een halve Eeuw A.N.W.B. 1883-1933, Schiedam 1934, S. 24, S. 40. Siehe auch die Beschreibung „Nederland is fietsenland“von Edo Bergsma, Ode aan de fiets, in: De Kampioen 45, 1928, S. 806; o.V., Onze wielerende natie, in: De Kampioen 45, 1928, S. 1197f. sowie J. v.d. Bergh, De fiets naast den auto - of de auto naast de fiets. Komt de fiets in gevaar? In ons land ... nooit, in: De Kampioen 45, 1928, S. $1373 \mathrm{f}$. Für das Jubiläumsjahr 1933 s.a. die Artikel o.V., 50 jaren van werken in het algemeen belang. Dankbaar Nederland, in: De Kampioen 50, 1933, S. 217-221; o.V. De plicht van Nederland jegens den A.N.W.B., in: De Kampioen 50, 1933, S. 877ff. u. E. Bergsma, De maatschappelijke waarde van het toerisme, in: De Kampioen 50, 1933, S. $1269 f$.

45 Vgl. H. te Velde, Gemeenschapszin en plichtsbesef. Liberalisme en Nationalisme in Nederland 1870-1918, Groningen 1992, S. 207-223. 
derländischen Geschichtsschreibung unter dem Stichwort der „beschavingsoffensief" verhandelt werden. ${ }^{46}$ Der bürgerliche Verband zielte darauf ab, die niederländische Bevölkerung im Sinne des Liberalismus zu verantwortungsbewussten Bürgern zu erziehen.

1908 gründete sich in Utrecht unter der Leitung des langjährigen Vorsitzenden des A.N.W.B., Edo Bergsma, die liberale Vereinigung „Tucht-unie“ (,Zucht-Union“). Die „Tucht-unie“ setzte sich zum Ziel, die von ihr diagnostizierte fortschreitende ,tuchteloosheid“ (,Zuchtlosigkeit“) in der Bevölkerung zu bekämpfen. ${ }^{47}$ Neben dem A.N.W.B. gehörten der ,Tucht-unie“ eine ganze Reihe weiterer liberaler Verbände an, darunter der ,Volksbond tegen Drankmisbruik“ (,Volksbund gegen Alkoholmissbrauch“), „Volksweerbaarheid“ (,Volkswehrbarkeit“), die „Maatschappij tot Nut van't Algemeen“ („Gesellschaft zum Nutzen der Allgemeinheit“), „Het Nederlandse Lied“ („Das Niederländische Lied“) und der „Nederlandse Bond voor Lichamelijke Opvoeding" („Der niederländische Bund für körperliche Erziehung"). Die „Tuchtunie" wurde so zu einem Amalgam von liberalen Vereinigungen und Verbänden, die sich die Aufgabe stellten, den niederländischen Staatsbürger und Bürger zu formen. Schlagwörter wie Zucht, Disziplin, Mannhaftigkeit und Wehrbarkeit, Verantwortungsgefühl und Vaterlandsliebe bildeten die Basis dieses liberalen Erziehungsauftrags. ${ }^{48}$

In diesem Sinne veröffentlichte Henri Meijer 1909 eine Serie von Artikeln in der Verbandszeitschrift De Kampioen über „De houding op de fiets“. 49 Meijer orientierte seine ideale Haltung auf dem Fahrrad vornehmlich an den Bedürfnissen des Touristen und verurteilte ausdrücklich die vornüber ge-

46 „Beschavingsoffensief“ heißt zu deutsch etwa „Zivilisierungsoffensive“. Den Begriff geprägt hatte vor allem B. Kruithof, De deugdzame natie. Het burgerlijk beschavingsoffensief van de Maatschappij tot Nut van't Algemeen tussen 1784 en 1860, in: ders., J. Noordman u. P. de Rooy (Hg.), Geschiedenis van opvoeding en onderwijs, 2. Aufl. Nijmegen 1983, S. 371-385. Zur „beschavingsoffensief“" als wissenschaftliches Analysetool s. J. Helsloot, Vermaak tussen beschaving en kerstening, Goes 1867-1896 (Publikaties van het P. J. Meertens-Institut, Bd. 24), Amsterdam 1995, S. 2-5; s.a. H.C.M. Michielse, Welzijn en discipline. Van tuchthuis tot psychotherapie: strategieën en technologieën in het sociaal beheer, Meppel 1989; P. de Rooy, Werklozenzorg en werkloosheidsbestrijding 1917-1940, Amsterdam 1979; A. de Regt, Arbeidersgezinnen en beschavingsarbeid. Ontwikkelingen in Nederland 1879-1940. Een historisch-sociologische studie, Meppel u. Amsterdam 1984. Energisch gegen den Begriff wandte sich W.W. Mijnhardt, Tot Heil van't Menschdom. Culturele genootschappen in Nederland, 1750-1815, Amsterdam 1987, S. 293f. Zu „beschaving“ als historischer Begriff s.a. R. Aerts u. W.E. Krul, Van hoge beschaving naar brede cultuur, 1780-1940, in: P. den Boer (Hg.), Beschaving. Een geschiedenis van de begrippen hoofsheid, heusheid, beschaving en cultuur, Amsterdam 2001, S. 213-254.

47 G.L. Janssen, De Tucht-unie. Haar ontstaan - wat zij deed - wat zij wil, Utrecht 1918.

48 Vgl. hierzu te Velde (wie Anm. 45), S. 218ff., und J. Bank u. M. van Buuren, 1900. Hoogtij van burgerlijke cultuur (Nederlandse Cultuur in Europese Context, Bd. 3), Den Haag 2000, S. 39.

49 H. Meijer, De houding op de fiets, Teile 1-3, in: De Kampioen 26, 1909, S. $147 \mathrm{ff} .$, S. $161 \mathrm{ff}$., S. 183ff. Übersetzung: „Die Haltung auf dem Fahrrad“. 
beugte Rennposition. ${ }^{50}$ Stattdessen befürwortete er einen ganz leicht gebeugten Oberkörper, der die Kontrolle über das Fahrrad gewährleiste und gymnastisch, federnd, locker und elegant sei.

Auf dem Fahrrad übte aber nicht nur der Radfahrer Kontrolle über die Maschine aus, umgekehrt zwang auch die Maschine den Radfahrer zu einem kontrollierten Körperverhalten. Gerade hiervon erhoffte sich der A.N.W.B. einen weiteren erzieherischen Effekt. So lobte Henri Meijer 1912, das Fahrrad sei wesentlich erfolgreicher im Kampf gegen den Alkohol als alle Ermahnungen und persönlichen Vorbilder. ${ }^{51}$ Nicht nur verzichte der ein oder andere auf seinen sonntäglichen Schnaps, um sich überhaupt ein Fahrrad leisten zu können, er lerne vielmehr auch, dass Alkohol die Kontrolle über den eigenen Körper auf dem Fahrrad stark beeinträchtige und dem Radfahrer viel Kraft raube. Auf diese Weise erfahre der „kleine Bürger“ am eigenen Leibe, wie wichtig Selbstkontrolle im Leben sei und erkenne im Alkohol einen gefährlichen Feind.

Mit dieser Propaganda knüpfte Meijer 1912 nahtlos an die ersten Veröffentlichungen über die Vorzüge des Fahrrads in den 1880er Jahren an. Noch vor der Jahrhundertwende hatte Bergsma das Fahrrad als ein „Werkzeug“ und den „Lehrmeister“ des niederländischen Bürgers bezeichnet. ${ }^{52}$ So wie das Fahrrad in jenen Jahren im männlichen Bürgertum wichtige Prinzipien wie Selbstkontrolle und Selbstständigkeit erneuern und bekräftigen sollte, so sollte es nun auch die erweiterten Kreise der Wahlberechtigten von der Notwendigkeit dieser bürgerlichen Tugenden überzeugen.

Der Erste Weltkrieg verstärkte diese Tendenzen und wirkte gewissermaßen als Katalysator für die Wahrnehmung der Niederlande als Fahrradland in der Zwischenkriegszeit. Die niederländische Gesellschaft blieb vom unmittelbaren Schock des Ersten Weltkriegs verschont, aber sie blieb von diesem Krieg nicht unberührt. ${ }^{53}$ Das Bewusstsein, neben der Katastrophe zu leben, war schon in den ersten Kriegsjahren deutlich vorhanden. Die Mobilisierung der Armee und der zeitweilige Zusammenbruch des Eisenbahnnetzes führten zu einer angespannten Lage. In dieser Zeit fügte der A.N.W.B. dem Radfahren eine weitere nationale Bedeutung hinzu. Umringt von Krieg führenden Nationen ermahnte De Kampioen seine Leser zu Ruhe und Gelassenheit. ${ }^{54}$ Dies sei nun die erste Bürgerpflicht, und der Verband riet dazu, angesichts des hektischen Auto- und Busverkehrs und der überfüllten, unzuver-

50 Ebd., S. 184.

51 H. Meyer, Het Rijwiel en de Drank, in: De Kampioen 29, 1912, S. $41 \mathrm{f}$.

52 Vgl. o.V., Aan den Lezer!, in: Maandblad 2, 1885, o. S.

53 Zum zunehmenden Interesse an der Bedeutung des Ersten Weltkriegs für die Niederlande siehe M.C. Brands, ,The Great War' die aan ons voorbijging. De blinde vlek in het historische bewustzijn van Nederland, in: M. Berman u. J.C.H. Blom (Hg.), Het belang van de Tweede Wereldoorlog, Den Haag 1997, S. 9-20; H. Binnenveld u.a. (Hg.), Leven naast de catastrofe. Nederland tijdens de Eerste Wereldoorlog, Hilversum 2001; P. Moeyes, Buiten schot. Nederland tijdens de Eerste Wereldoorlog 1913-1918, Amsterdam u. Antwerpen 2001.

54 O.V., Wij moeten, in: De Kampioen 31, 1914, S. $727 f$. 
lässigen Eisenbahn besser das Fahrrad zu nehmen. ${ }^{55}$ Der Kriegsausbruch habe viele Menschen schockiert und ihre Stimmung nachhaltig niedergedrückt, daher sei es nun äußerst wichtig, nicht die Nerven zu verlieren und die eigene Ruhe wieder zu finden. Die regelmäßige, ruhige Tretbewegung auf dem Fahrrad könne hier entscheidend mithelfen, die Stimmung zu verbessern und das geistige Gleichgewicht zu stärken. Während der Kriegsjahre intensivierte der Verband seine Arbeit für den Radtourismus im eigenen Land. Dies war einerseits eine praktische Maßnahme, da der Automobilverkehr in dem zusehends von der Seeblockade betroffenen Land zum Erliegen kam und Auslandsreisen kaum noch möglich waren. Zugleich entsprach es aber auch der langjährigen Politik des A.N.W.B., das Fahrrad als Mittel einzusetzen, um die Liebe zum eigenen Land zu fördern. So fallen die Anfänge des Aufbaus eines eigenständigen nationalen Radfahrwegenetzes in den Niederlanden in den Ersten Weltkrieg. Innerhalb von wenigen Jahren entstand ab 1914 eine ganze Reihe so genannter „Rijwielpadverenigingen“ („Radfahrwegevereinigungen“) lokaler Vereine, die unter der Ägide des A.N.W.B. die Anlage von Radfahrwegen vor Ort vorantrieben. ${ }^{56}$ Von Anfang an waren diese neu angelegten Radfahrwege fester Bestandteil des national integrativen Tourismuskonzeptes des A.N.W.B. Bereits 1915 veröffentlichte der Verband eine Broschüre über Rijwieltochten over rustige wegen. Das Fahrrad, so die Einleitung, habe den Niederländern ihr eigenes Land und dessen Schönheit näher gebracht. ${ }^{57}$ Leider jedoch würden sich viele Radfahrerinnen und Radfahrer bei ihren Touren auf die großen Straßen konzentrieren, deren Benutzung in den letzten Jahren infolge der Motorisierung nicht angenehmer geworden sei. Den wenigsten sei bekannt, „dat men de intieme schoonheid van ons land het best leert kennen op de kleinere binnenwegen, en het allerbest op de rijwielpaden door bosch en heide" .58

Der A.N.W.B. überwachte und koordinierte das Ineinandergreifen der lokalen und regionalen Initiativen in ein nationales Ganzes. Der Verband sorgte dafür, dass die Wege so angelegt wurden, dass sie über Gemeinde-

55 O.V., Per fiets, in: De Kampioen 31, 1914, S. 773 f.

56 In den Akten des A.N.W.B. sind folgende Radfahrwegevereine aus der Zeit des Ersten Weltkriegs verzeichnet: „Gooi- en Eemland“(1914), „Drenthe“(1916), „E.M.O.“(Eindhoven met Omstreken, gegründet 1917), „Noord Veluwe“ (1917), „Twenthe“ (1917), „Zuid-Holland“ (1917), „U.M.O.“ (Utrecht met Omstreken, 1918), H.E.R.M.O. ('s Hertogenbosch met Omgeving, 1918). Daneben gab es auch Bemühungen zur Gründung von „Rijwielpadverenigingen“ in Friesland (1917), Midden-Limburg (1918) und im Zuidwesthoek von Overijssel (1919). Zu den späteren Gründungen zählen: „B.R.O.G.“ (Bond van Rijwielpadvereenigingen in Oosterlijk Gelderland, gegründet 1930), „Noord Kennemerland“ (1920) und „Walcheren“ (1926). Ohne Gründungsdatum: „West Veluwe“ und „Oost Gelderland“",Zwolle“. Vgl. A.N.W.B. Archief 171-181, Rijwielpadverenigingen.

57 A.N.W.B. Toeristenbond voor Nederland (Hg.), Rijwieltochten over rustige wegen, zusammengestellt von G.A. Pos, Den Haag 1915, S. 2. Übersetzung des Buchtitels: „Radfahrausflüge über ruhige Wege“.

58 Ebd. Übersetzung: ,dass man die intime Schönheit unseres Landes am besten auf den kleineren Wegen und am allerbesten auf den Radfahrwegen durch Wald und Heide kennenlernt". 
grenzen hinweg aneinander anschlossen und führte ab 1919 die Radfahrwege über ein nationales Wegweisersystem zusammen. Diese Wegweiser in Pilzform wichen in Gestaltung und Form deutlich von den Wegweisern ab, die der A.N.W.B. für die großen Wege vorgesehen hatte. Die Leistungen der privaten Initiativen auf dem Gebiet des Radfahrwegebaus waren beachtlich: 1938 schätzte die „Federatie van Nederlandsche Rijwielpadvereningen“ die von den Vereinen angelegten Radfahrwege auf 2.500 Kilometer. ${ }^{59}$ Demnach wäre die Gesamtstrecke der frei liegenden, unter der Beratung und Koordination des A.N.W.B. privat angelegten Radfahrwege nur geringfügig niedriger als die Zahl der Radwege, die in Obhut von Reich und Provinzen (insgesamt 2.664 Kilometer) angelegt worden waren. Der A.N.W.B. hatte sich erfolgreich als „Systembauer“ bei der Gestaltung des modernen StraBenverkehrs in den Niederlanden etabliert. ${ }^{60}$ Zugleich hatte der Verband mit diesem Radfahrwegenetz noch einmal seine Vorstellung vom korrekten Gebrauch des Fahrrads untermauert. Auf dem Fahrrad sollten niederländische Bürger ihr eigenes Land kennen lernen. Ein korrekter Gebrauch beinhaltete auch die Bevorzugung bestimmter Fahrradmodelle und legte ein entsprechendes Design der Fahrräder nahe.

\section{Auf der Suche nach dem „Holland-Rad“": Die Entwicklung der nieder- ländischen Fahrradindustrie}

Trotz der zunehmenden Zahl von Radfahrern im Land und der Aneignung des Fahrrads in einem nationalen Kontext seitens des niederländischen Radfahrerverbands A.N.W.B. gewann die niederländische Fahrradindustrie erst allmählich an Bedeutung. In der Zeit vor dem Ersten Weltkrieg war die Niederlande der größte Importeur von Fahrrädern aus England ${ }^{61}$ Verschiedentlich beklagte der Chefredakteur der Verbandszeitschrift des A.N.W.B. den hohen Import an Fahrrädern, obwohl es den niederländischen Fahrrädern an nichts mangele, und bezichtigte 1908 die ausländischen Hersteller gar der unlauteren Konkurrenz. Sie würden ihre Handelsvertreter mit überhöhten Provisionen zum Verkauf ihrer Produkte anstacheln. Dabei übersah er geflissentlich, dass die niederländischen Produzenten aufgrund ihrer veralteten Produktionsweisen gerade im Billigsektor kaum mit der ausländischen Konkurrenz mithalten konnten. ${ }^{62}$

59 A.N.W.B. Archief 177, Rijwielpadverenigingen, Vergadering van de Federatie van Nederlandsche Rijwielpadvereenigingen, gehouden te Zeist, in Hôtel Hermitage, op 10 Juni 1938, S. 14.

60 Vgl. zum A.N.W.B. als „Systembauer“, allerdings mit einem etwas einseitigen Fokus auf den motorisierten Verkehr: G.P.A. Mom, J.W. Schot u. P.E. Staal, Werken aan mobiliteit: de inburgering van de auto, in: J.W. Schot u.a. (Hg.), Techniek in Nederland in de Twintigste Eeuw, Bd. 5: Transport en communicatie, Zutphen 2002, S. 45-74, hier S. $62 \mathrm{ff}$.

61 Meijer (wie Anm. 12).

62 Vgl. H. Meijer, Onedele concurrentie, in: De Kampioen 25, 1908, S. $405 \mathrm{ff}$. 
Deutsche Exporteure wie beispielsweise die Firma Dürkopp in Bielefeld verfügten über eine breite Palette an Fahrradmodellen, vom Tourenrad über den Halbrenner, den Renner und das Straßenrennrad. Dürkopp bot 1907 sowohl für das Tourenrad als auch für den Halbrenner eine Variante mit nach vorn abfallendem Rahmen an. ${ }^{63}$ Daneben gab es das traditionelle elegante Tourenrad mit geradem Rahmen, das sich in seiner Gestalt seit 1898 kaum verändert hatte. ${ }^{64}$

In den Niederlanden hingegen offenbaren die Kataloge der Firma Burgers aus Deventer eine geradezu gegenläufige Entwicklung: Wurden 1898 mehrere Modelle mit nach vorn abfallendem Rahmen angeboten, besaßen 1915 alle angebotenen Modelle einen geraden Rahmen. Die Rennmodelle waren gänzlich entfallen. ${ }^{65}$ Eine Reaktion auf das Verbot von Straßenrennen im Land? Oder kauften die niederländischen Konsumenten lieber ausländische Rennfabrikate? Auf diese Fragen ist nur schwer eine Antwort zu finden, da über die konkreten Absatzzahlen kaum etwas bekannt ist. Auffällig ist indes, dass sowohl deutsche als auch niederländische Hersteller in der Zeit bis zum Ersten Weltkrieg Tourenräder anboten, die in ihrer Form dem heutigen Holland-Rad entsprachen (s. Abbildung 2).

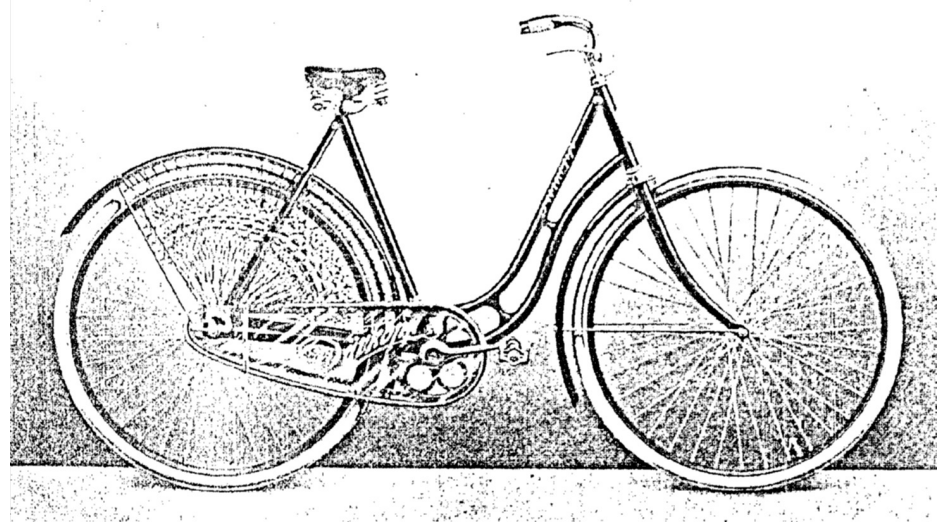

Abb. 2: Dürkopps Diana 62 von 1907. Quelle: Stadtarchiv Bielefeld, Bestand 210,5/Dürkopp Adler, Nr. 813: Katalog der Bielefelder Auto-, Fahrrad- und Nähmaschinenfabrik Dürkopp von 1907 [Kopie], S. $16 f$.

63 Vgl. Bielefelder Maschinen-Fabrik vormals Dürkopp \& Co., Illustrierter Katalog über Dürkopp-Fahrräder, Bielefeld 1907, S. 24f., S. 30f.

64 Vgl. Bielefelder Maschinen-Fabrik vormals Dürkopp \& Co., Dürkopp's Diana, allen voran, Bielefeld 1898.

65 Eerste Nederlandsche Rijwielfabriek, Directeur H. Burgers, Deventer (Hg.), Prijscourant 1897 und Burgers E.N.R. Rijwielen, Prijscourant 1915. 
In den 1920er Jahren steigerte sich die niederländische Fahrradproduktion kontinuierlich. Von 49.300 Stück im Jahr 1919 stieg sie auf 134.700 Fahrräder im Jahr 1924. Ab Mitte der 1920er Jahre konnte die Produktion noch einmal beträchtlich bis auf 405.000 Stück im Jahr 1929 angehoben werden. ${ }^{66}$ Dennoch gelang es den niederländischen Herstellern erst Ende der 1920er Jahre, den eigenen Markt vollständig abzudecken. Erich Eicker konstatierte 1929 im Rahmen einer Dissertation über die deutsche Fahrradindustrie eine Sättigung des niederländischen Markts in Bezug auf den Export deutscher Räder, das Nachbarland sei jedoch weiterhin der aufnahmefähigste Exportmarkt für die deutsche Teile- und Zubehörteilefabrikation. ${ }^{67}$ In einer Analyse der niederländischen Fahrradproduktion in den Economisch-Statistischen Berichten beklagte van Hoogstraten 1930, dass diese neben einigen großen Betrieben immer noch aus zu vielen kleineren Betrieben bestünde. ${ }^{68}$ Die in diesen Betrieben stärker zum Einsatz kommende menschliche Arbeitskraft verteuere aber die Produktion, was den geringen Export von Fahrrädern aus den Niederlanden erkläre.

Neben den Fahrradfabriken existierten zunächst zahlreiche Fahrradmontagebetriebe. 1928 zählte das statistische Zentralbüro des Landes bei einer Mindestproduktion von 500 Fahrrädern im Jahr mehr Montagebetriebe (49) als Fabriken (40).$^{69}$ Bei insgesamt steigenden Produktionszahlen konsolidierte sich die Industrie im Verlauf der 1930er Jahre. 1937/38 waren in der Statistik insgesamt nur noch 35 Fabriken aufgeführt, Montagebetriebe tauchten nicht mehr auf. Deren Produktionsleistungen hatten zwischen 1933 und 1937 bereits deutlich abgenommen, so dass die insgesamt steigende Anzahl produzierter Fahrräder vor allem auf die großen Fabriken zurückzuführen war. Insbesondere in der Rahmenherstellung erhöhte sich die Stückzahl von 45.000 im Jahr 1935 auf 75.000 im Jahr 1937. Dennoch importierten die Niederlande auch in den 1930er Jahren nach wie vor zwei Drittel aller Fahrradteile aus Deutschland. ${ }^{70}$ Von einer nationalen Produktion bei der Fahrradherstellung zu sprechen, ist also mit Blick auf das fertige Produkt durchaus irreführend. Die industriell gefertigten Fahrräder in den Niederlanden bestanden aus einer Vielzahl von

66 Vgl. o.V. Rijwielfabrieken, 1925, in: De Kampioen 44, 1927, S. 270 u. o.V. Een statistiek van onze Nederlandse rijwielproductie, in: De Kampioen 47, 1930, S. $1209 \mathrm{f}$.

67 Erich Eicker, Der Aufbau der deutschen Fahrradindustrie, Inaugural-Dissertation zur Erlangung der Doktorwürde der Wirtschafts- und Sozialwissenschaftlichen Fakultät der Universität Köln, Köln 1929, S. 53. Vgl. hierzu auch o. V., Toenemende populariteit der fiets in Nederland, in: De Kampioen 45, 1928, S. 310. Zum deutschen Export in die Niederlande vgl. auch Otto E. Seyfert, Die deutsche Fahrradindustrie. InauguralDisseration zur Erlangung der Doktorwürde der hohen philosophischen Fakultät der Großherzoglich Badischen Ruprecht-Karls Universität in Heidelberg, Borna, Leipzig 1912, S. 92 u. 94.

68 S. van Hoogstraten, De Rijwielindustrie in 1929, in: Economisch-Statistische Berichten 15,1930 , S. $680 \mathrm{f}$.

69 Zitiert nach o.V., Die Fahrradwirtschaft Hollands, in: Der Radmarkt 2561, 1940, S. 6f., hier S. 6.

70 Ebd. 
Einzelteilen unterschiedlicher Herkunft. Es existierten zwischen den Fabriken keinerlei Absprachen über Rahmenbau und Farbe. Es gab keine Absprachen und auch keine professionellen Designer des Holland-Rads. ${ }^{71}$

Der Eindruck, dass die niederländischen Fahrradproduzenten mit ihrer Produktpalette auch auf die gesetzlichen Vorgaben reagierten, bestätigt sich für die 1930er Jahre: In den Katalogen finden sich kaum Rennräder. ${ }^{72}$ Zugleich griffen sie in ihrer Werbung auf eine Ikonographie des Fahrrads in den Niederlanden zurück, die maßgeblich vom A.N.W.B. geprägt worden war. So bewarb Fongers aus Groninger seine Fahrräder in den 1930er Jahren mit einem Werbeplakat, das auch vom Radfahrerverband hätte stammen können. Auf einer großen Landkarte der Niederlande waren Radfahrer zu sehen, die das Land bereisten. Der Slogan des Plakats: „Wenn Du, Radfahrer, Urlaub hast, sind die gesamten schönen Niederlande Dein Terrain ..." (s. Abbildung 3).

Abb. 3: Fongers Rijwielreklame aus den 1930er Jahren. Quelle: Groninger Archieven 1300, Archieven van de Groninger Rijwielenfabriek A. Fongers, Nr. 234.

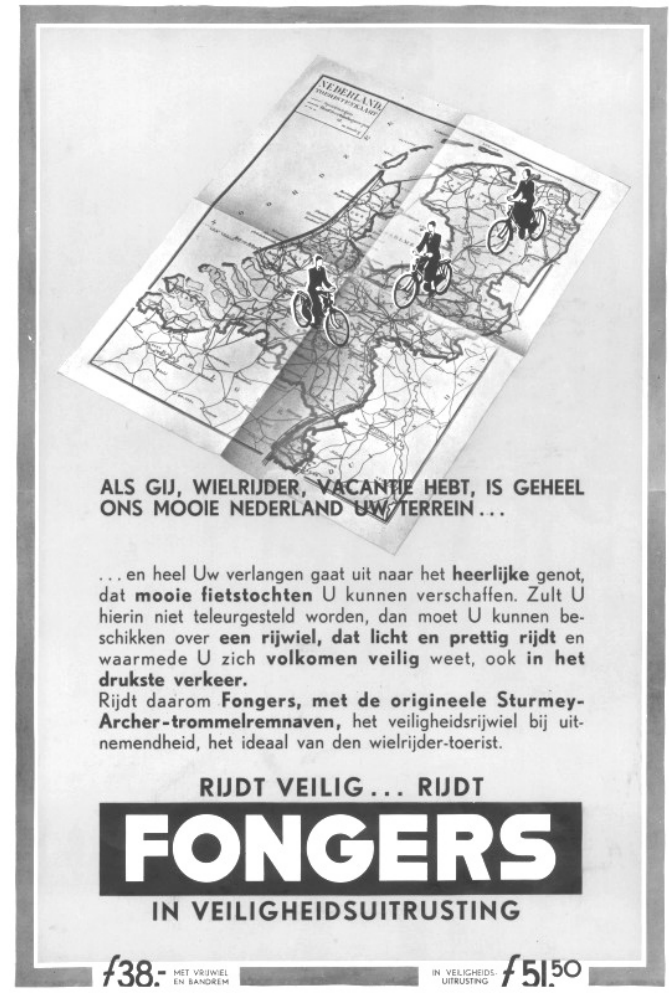

71 Die Fahrradproduktion gehörte somit zu den Bereichen, in denen eine Professionalisierung des Design-Berufs zu Beginn des 19. Jahrhunderts noch nicht sehr weit vorgeschritten war. Zur zunehmenden professionellen Gestaltung von Technik s. Gert Selle, Geschichte des Design in Deutschland, Frankfurt a.M. 2007, S. 36ff.

72 Vgl. z.B. Burgers E.N.R. Prijscourant von 1932; Fongers Prijscourant 1930 jeweils ohne Rennrad im Angebot. Dagegen Prijscourant Gazelle von 1932 mit einem Rennrad. 
In den 1930er Jahren gab es Ansätze, den Fahrrädern in den Niederlanden einen nationalen Stil zuzuschreiben. Ein Diplomingenieur namens Grote suggerierte in einem Artikel in der deutschen Zeitschrift Radmarkt von 1939, die Rahmenform des Fahrrads in Deutschland zu überdenken und sich hierbei ein Vorbild an den niederländischen Fahrrädern zu nehmen. In Holland werde viel mehr geradelt als in Deutschland, und dort wundere man sich ,über die krumme Haltung, in der bei uns gefahren wird“. ${ }^{73}$ Gegen eine ,,solche Verallgemeinerung" wandte sich ein anderer Autor energisch. ${ }^{74}$ Er hob hervor, dass sich die Rahmen der Räder nach ihrem Zweck unterschieden; dies führe zur unterschiedlichen Position der Fahrer auf Stadt- und Rennrädern. In den Niederlanden überwiege die Anzahl der Gebrauchs- und Stadtfahrräder, auf denen im Allgemeinen mit einer stärker aufrechten Haltung gefahren werde. Von einem nationalen Stil in der Produktion könne daher nicht die Rede sein. Wenn überhaupt, dann gäbe es unterschiedliche nationale Gebrauchsweisen. Mit dieser Argumentation bezweifelte der Autor, ob sich beim industriell gefertigten Massenprodukt Fahrrad nationale Produktionsweisen unterscheiden lassen. Sehr wohl unterschied er indes nationale Konsumgewohnheiten. Die Nutzer des Fahrrads, die Konsumenten, wurden mit ihrer Bevorzugung bestimmter Rahmenkonstruktionen, die im besonderen Maße ihren Gebrauchsmustern mit dem Fahrrad entsprachen, zu den eigentlichen Konstrukteuren eines niederländischen Fahrrad-Designs.

\section{Aneignung, Technik und Design: Schnittpunkt Holland-Rad}

Das Holland-Rad ist die materielle Umsetzung der Bedeutung und der adäquaten Nutzungsweisen des Fahrrads in den Niederlanden. Das ,,anonyme Design" des Holland-Rads war Ausdruck eines nationalen Aneignungsprozesses, bei dem bestimmte Nutzungsformen in einem gesellschaftlichen Aushandlungsprozess favorisiert und auf diese Weise auch bestimmte Fahrradformen geprägt worden waren. Das Holland-Rad resp. „omafiets“, wie es bis heute von deutschen und niederländischen Produzenten angeboten wird, beruht auf einer Rahmenkonstruktion, die bereits seit der Jahrhundertwende von deutschen und englischen Herstellern offeriert wurde. Mit der nationalen Aneignung des Fahrrads seitens des niederländischen Bürgertums entstand seit der Jahrhundertwende die Vorstellung eines typisch niederländischen Fahrradgebrauchs, lange bevor die inländische Fahrradproduktion überhaupt eine Bedeutung auf dem heimischen Markt einnehmen konnte. Vor dem Hintergrund der Umwälzungen in der niederländischen Politik propagierte der A.N.W.B. das Fahrrad als Mittler liberaler Werte, die durch die Körperübung des Radfahrens gefördert und trainiert werden sollten. Der Verband hatte sich frühzeitig gegen Radrennen gewandt und war in dieser

73 Vgl. Grote, Das Fahrrad bei uns und in Holland, in: Der Radmarkt 2510 (1939), S. 17.

74 Vgl. o.V., Das Fahrrad bei uns und in Holland, in: Der Radmarkt 2520 (1939), S. 5f. 
Haltung von der Gesetzgebung im Land unterstützt worden, die 1905 die Straßenrennen verbot. Stattdessen hatte der Verband das Bereisen des eigenen Landes auf dem Fahrrad mit allen Mitteln vorangetrieben. Die mit dem Radtourismus verbundene, aufrechte Sitzhaltung verband sich aufs engste mit dem liberalen Erziehungsprogramm der niederländischen Bürger. Als einflussreicher Konsumentenverband prägte der A.N.W.B. eine bestimmte Praxis des Radfahrens, die wiederum förderte den Gebrauch bestimmter Fahrradmodelle, namentlich der Stadt- und Tourenfahrräder. Auf diese Weise entstand die Vorstellung von einem typisch niederländischen Fahrraddesign, das maßgeblich von den Nutzern des Fahrrads geprägt wurde. Das ,anonyme Design" des Holland-Rads demonstriert somit eindrucksvoll die große Bedeutung der Konsumenten als Ko-Konstrukteure und Ko-Designer.

Das Holland-Rad verdeutlicht zugleich auch die Konvergenz von Technik und Design bei industriell gefertigten Serienprodukten des frühen 20. Jahrhunderts. Der nach vorne abfallende Rahmen trug sowohl eine aerodynamische und mithin (körper-)technische Komponente wie auch DesignAspekte in sich. Historiker/innen tun daher gut daran, beide Aspekte gemeinsam und auch im Kontext der Nutzer/innen und Nutzungsformen zu betrachten. Dies bedeutet, dass die Technikgeschichte auch nicht davor zurückschrecken sollte, im Zuge einer Betrachtung der Wechselwirkungen zwischen Produktion und Konsumtion auch ,weiche Faktoren“ wie beispielsweise den Schwung der Rahmengebung zu berücksichtigen. Umgekehrt profitiert eine sozial- und kulturgeschichtlich orientierte Designgeschichte gerade im Falle des ,anonymen Designs“von den Überlegungen und Ansätzen aus der Technikgeschichte, die Rolle der Konsumenten als Ko-Designer stärker in die Analyse mit einzubeziehen.

Schließlich gibt es auch noch die Geschichte des Holland-Rads auf dem deutschen Markt der Gegenwart. Während das nostalgische Stadtrad technisch mit SRAM-Gangschaltung und Trommelbremsen den gegenwärtigen Ansprüchen zumindest leicht angepasst wird, stellt es in der Rahmenkonstruktion ein Design dar, das sich an den Fahrradmodellen der Jahrhundertwende orientiert. Dass es sich hierbei aber eben nicht um ein typisch niederländisches Design handelt, bleibt den meisten Nutzern vermutlich ebenso verborgen wie die Tatsache, dass sie mit dem vermeintlichen Holland-Rad ein Erziehungsmittel des niederländischen Liberalismus erwerben. So wandelte sich zwar nicht das Design, wohl aber änderte sich die Bedeutung des Stadtrads im Laufe des Jahrhunderts in den Niederlanden und in Deutschland.

Anschrift der Verfasserin: Dr. des. Anne-Katrin Ebert, Technisches Museum Wien, Mariahilfer Str. 212, 1140 Wien, E-Mail: anne.ebert@tmw.at 
